\title{
Performance Evaluation of Wide-Angle Ultrathin Microwave Metamaterial Absorber with Polarization Independence
}

\author{
Kanwar Preet Kaur ${ }^{* 1}$ and Trushit Upadhyaya ${ }^{1}$ \\ ${ }^{1}$ Department of Electronics \& Communication Engineering, Chandubhai S. Patel Institute of Technology, Charotar University \\ of Science \& Technology, Changa, Anand-388421, Gujarat, India \\ *corresponding author, E-mail: kanwarpreet27@gmail.com
}

\begin{abstract}
A novel dual-band wide-angle ultra-thin absorber composed of dual closed-concentric ten-point stars with polarization independence is presented in this article. The ten-point stars formed resonators are engraved on low cost FR4 substrate. The EM absorber performance is studied in terms of electrical and physical parameters. The polarization independence is practically demonstrated using waveguide measurement method. The measured results are in good agreement with simulated results. The absorbance values of $99.37 \%$ and $97.18 \%$ are achieved at $2.13 \mathrm{GHz}$ and 2.52 $\mathrm{GHz}$, respectively. The presented absorber design is wideangle RF absorber with high absorbance values of about $80 \%$ up to $60^{\circ}$ under TE mode and $87 \%$ up to $75^{\circ}$ of oblique incidence angle for TM mode excitations. The experimental results demonstrate the absorption effectiveness of the proposed design to be utilized in RF energy harvester modules for capturing ambient UMTS band signals and in RF absorber applications for $\mathrm{S}$ band radar systems. The presented absorber unit cell is ultrathin and compact with the thickness and size less than $\lambda / 56 \mathrm{~mm}$ and $\lambda / 5 \mathrm{~mm}$, respectively, at lowest absorption frequency.
\end{abstract}

\section{Introduction}

Owing to the exceptional properties of metamaterials (MTM), introduced by Victor Veselego in 1968 [1], many researches based on MTM have been carried out in recent past. The most significant properties of MTMs are the negative permittivity, negative permeability and negative refractive index $[2,3]$ which can be adjusted to an optimized value by merely altering the geometrical parameters. Because of the compactness provided by MTMs, especially in RF domain, they are enormously utilized in the absorber structures. The first metamaterial absorber (MMA) was proposed and practically verified by N. I. Landy in 2008 [4] with the absorption value of $96 \%$ at $11.48 \mathrm{GHz}$ and full width at half maximum (FWHM) of $4 \%$. Subsequently, many MMAs have been reported and investigated with the distinct characteristics from microwave to infrared bands [5,6]. MMAs of the following significant characteristics have been reported so far; dualband $[7,8]$, triple-band $[9,10]$, quad-band $[11,12]$, pentaband [13], bandwidth-enhanced [14], wide-band [15, 16], wide-angle $[17,18]$ and polarization insensitivity $[19,20]$ to accomplish various RF applications. The bandwidth of MMAs can be increased extensively by using layered resonator structures and tuned to the specified value as reported in [21-23]. The drawback of layered structures is that they may not be suitable for ultra-thin broadband applications requiring the total thickness below $\lambda_{0} / 4$ where $\lambda_{0}$ represents the absorption wavelength. Numerous ultrathin compact MMAs have been reported having multiband/broadband response without using stacking of unit cells [24-26]. Some researchers outlined ultra-thin absorbers with the stacking technology achieving broadband absorption at terahertz region [27]. Thus there is still requirement of simple, compact polarization independent MMAs working in L-band and lower S-band.

In this article, a wide-angle microwave MMA with the polarization independence has been presented. RF absorption performance of the designed structure is evaluated by varying various electrical as well as physical parameters for the proper selection of unit cell design based on the requirement of bandwidth and frequency. The proposed MMA is numerically analysed by using finite element method (FEM) based 3D commercial full wave electromagnetic field high frequency structure simulator (HFSS) solver. Numerically computed absorption values of the suggested MMA are obtained as $99.37 \%$ and $97.18 \%$ at $2.13 \mathrm{GHz}$ and $2.52 \mathrm{GHz}$, respectively. Proposed MMA structure has wide incidence angle of about $60^{\circ}$ under TE mode excitation with the absorption of $80 \%$ and $75^{\circ}$ for TM mode excitation with the absorption value of $87 \%$. Furthermore, MMA design has been studied for the polarization independence and the experimental demonstration is performed by using waveguide measurement technique [28]. For the practical verification of the polarization independence, different MMA structures are fabricated in various orientation angles. A good agreement between the numerically computed results and measured results makes the proposed MMA structure a good candidate for capturing ambient UMTS band signals in the RF energy harvester modules and RF absorber in Sband radar system applications. In this paper, Section 2 describes the unit cell design with the brief details of the absorption principle. Section 3 presents the numerical computation and related discussion of results. In Section 4, 
the numerical MMA model is investigated for the various electrical and physical parameters as numerical parametric studies. Section 5 describes the measurement setup along with the experimental results. The concluding remarks are conducted in Section 6.

\section{Proposed MMA Unit Cell Design}

The unit cell of proposed wide-angle MMA is composed of dual concentric-closed ten-point stars engraved on a low cost metal backed FR4 substrate. The perspective view of the proposed unit cell model and the fabricated absorber prototype are shown in Fig. 1.

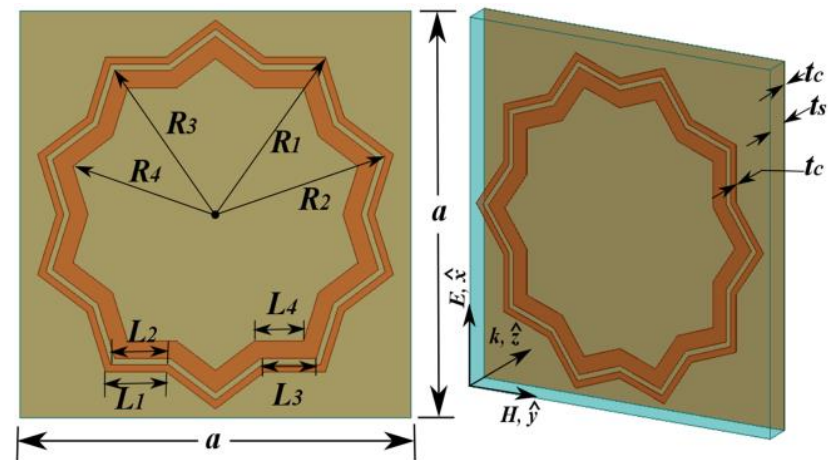

(a)

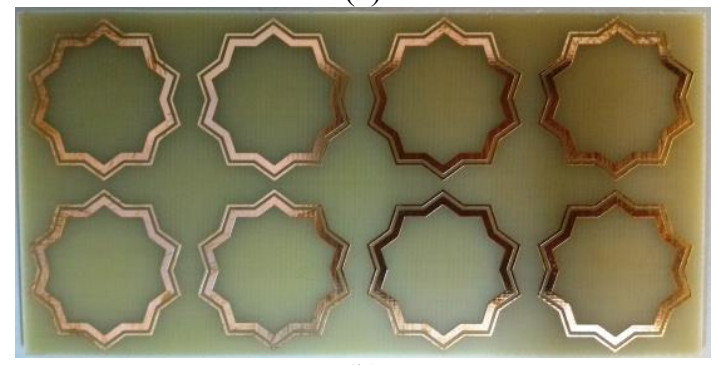

(b)

Figure 1: (a) Perspective view of MMA unit cell model and (b) fabricated prototype.

Ten-point star resonator geometry is decided due to the resulting increased electrical length which reduces the absorption frequency, which is obtained in large scale unit cells operating at the low absorption frequency with high absorbance in large oblique angle of both TE-mode and TM-mode excitations. The substrate metal is the copper with the conductivity $(\zeta)$ of $5.8 \times 10^{7} \mathrm{~S} / \mathrm{m}$ having the thickness $\left(\mathrm{t}_{\mathrm{c}}\right)$ of $0.035 \mathrm{~mm}$ much higher than the skin depth at the frequency band of interest. The concentric ten-point star resonator and back metal plate are separated by the substrate of height $\left(t_{\mathrm{s}}\right) 2.4 \mathrm{~mm}$, having relative dielectric constant $\left(\varepsilon_{\mathrm{r}}\right)$ of 4.4 and loss tangent $(\tan \delta)$ of 0.02 . The optimized parameters of the unit cell structure are as follows: $a=27.4 \mathrm{~mm}, L_{1}=4.26 \mathrm{~mm}, L_{2}=4.06 \mathrm{~mm}, L_{3}=$ $3.9 \mathrm{~mm}, L_{4}=3.41 \mathrm{~mm}, R_{1}=13.1 \mathrm{~mm}, R_{2}=12.5 \mathrm{~mm}, R_{3}=$ $12 \mathrm{~mm}$, and $R_{4}=10.5 \mathrm{~mm}$. The presented MMA unit cell is ultrathin with the thickness and size less than $\lambda / 56 \mathrm{~mm}$ and $\lambda / 5 \mathrm{~mm}$, respectively, at the lower absorption frequency. One of the most significant parameter that defines the effectiveness of an RF absorber is its absorbance value which is related to the reflectance, $R(\omega)$, and the transmittance, $\mathrm{T}(\omega)$, by the following relation.

$$
A(\omega)=1-R(\omega)-T(\omega)
$$

$\mathrm{R}(\omega)$ and $\mathrm{T}(\omega)$ are directly related with the return loss and the insertion loss in the form of $R(\omega)=\left|S_{11}(\omega)\right|^{2}$ and $T(\omega)=\left|S_{21}(\omega)\right|^{2}$.

\section{Numerical Computation and Result Discussions}

A MMA array of $2 \times 4$ unit cells having the periodic arrangement of $27.4 \mathrm{~mm}$ is simulated using HFSS electromagnetic field solver. The periodic boundary conditions are imposed through Floquet port excitation with the electric field and magnetic field positioned along $\mathrm{X}$-axis and Y-axis, respectively. The incoming EM wave is applied normal to the surface of the structure (along $\mathrm{Z}$-axis). Due to the back metal plate, negligible transmittance is obtained from MMA structure in comparison to the absorption and reflectance parameters. Thus, the absorbance value is calculated by substituting the return loss values in Eq. (1). The comparative numerically computed results of the absorbance, reflectance and transmittance are depicted in Fig. 2(a) along with the absorption response for the various polarization angles $(\phi)$ under the normal incidence of EM waves at constant oblique angle $(\theta)$ in Fig. 2(b).

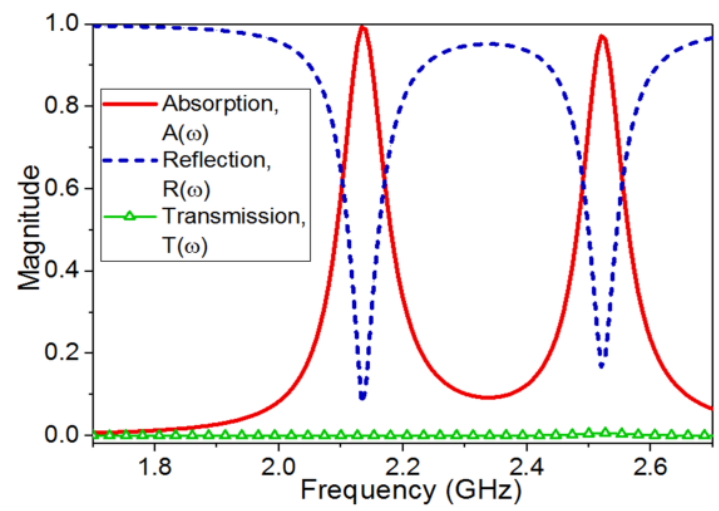

(a)

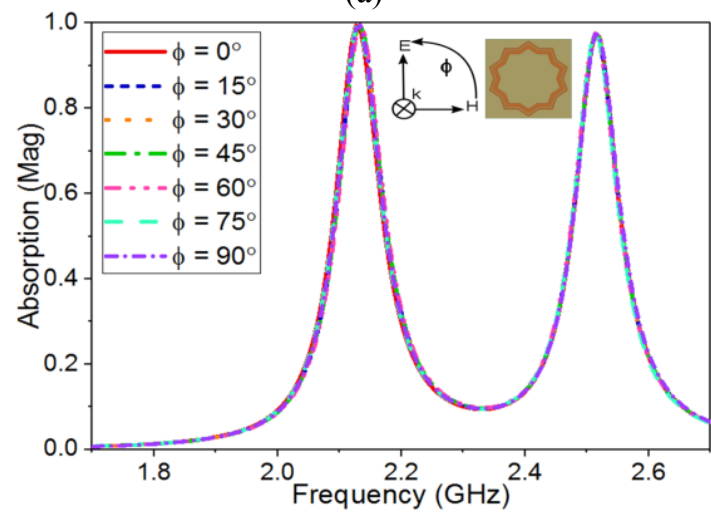

(b)

Figure 2: (a) Comparison of numerically calculated EM wave absorption, reflection and transmission properties and (b) absorption response at various polarization angles $(\phi)$ under normal incidence of EM wave. 
It is observed that the proposed structure is highly polarization insensitive owing to the foldable symmetrical unit cell design. The variation of the incidence angle $\theta$ under TE- and TM-mode excitations for the fixed $\phi$ angle has been studied further as shown in Fig. 3. In the case of TE-mode, the electric field direction is kept same under the variation of the magnetic field and wave propagation vector by the incidence angle $\theta$ as illustrated in Fig. 3(a). Whereas, in the case of TM-mode, the electric field and wave propagation vector are varied by the incidence angle $\theta$ keeping the direction of magnetic field in the same direction as illustrated in Fig. 3(b). From Fig. 3, it is deduced that the proposed MMA design is a wide-angle absorber with high absorbance value of $80 \%$ up to $60^{\circ}$ under TE mode whereas the absorbance value is as high as $87 \%$ up to $75^{\circ}$ of oblique incidence angle for TM mode.

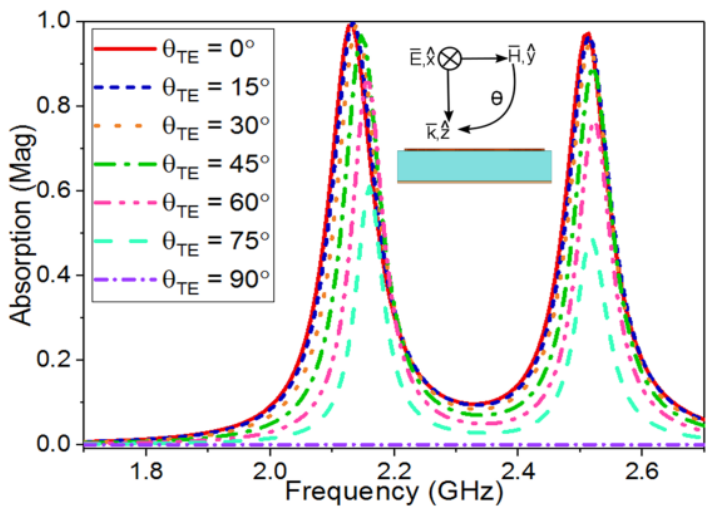

(a)

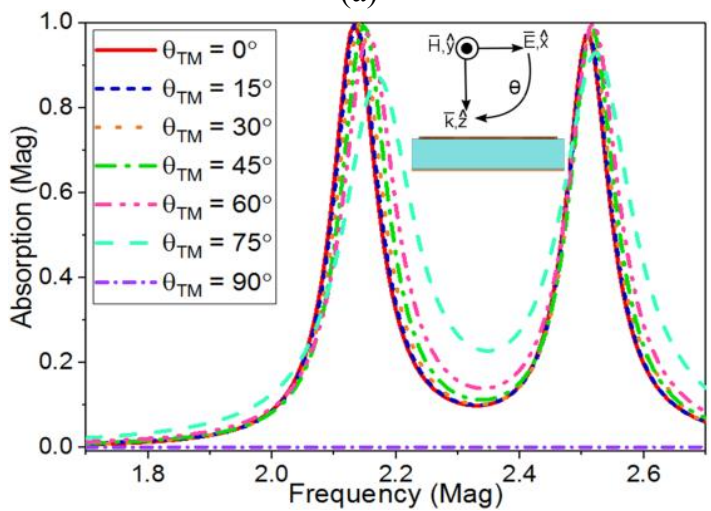

(b)

Figure 3: Simulated absorption response at various oblique angles $(\theta)$ under fixed polarization for (a) TE configuration and (b) TM configuration.

\section{Numerical Parametric Studies}

To analyse the geometric and material parameters affecting the absorption value and absorption frequencies of incoming EM wave, MMA has been numerically computed for the various physical and electrical parameters. The absorption response with the variations in the dielectric thickness $\left(t_{s}\right)$ is shown in Fig. 4(a). It is noted that the magnitude of absorption decreases gradually with the improvement in the bandwidth as the substrate height increases. The substrate height of $4.8 \mathrm{~mm}$ indicates the lower absorption peak with the wider bandwidth. The proposed MMA is further studied under the variation of relative dielectric constant $\left(\varepsilon_{\mathrm{r}}\right)$ as shown in Fig. 4(b).

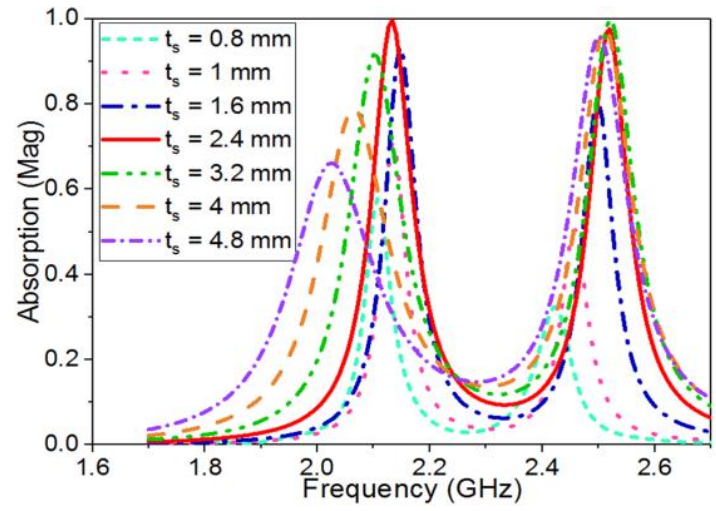

(a)

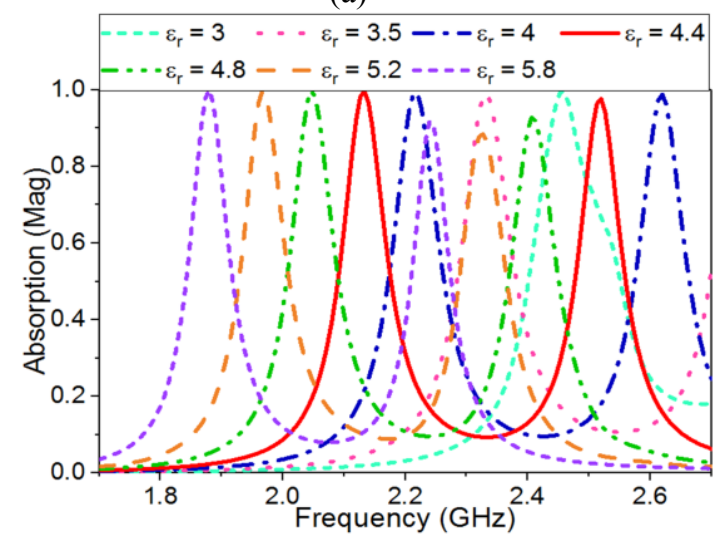

(b)

Figure 4: Simulated absorption response for various (a) Substrate Height $\left(t_{s}\right)$ and (b) Relative dielectric constant $\left(\varepsilon_{\mathrm{r}}\right)$.

As the relative permittivity of substrate is increasing, the absorption frequency band is shifted towards the lower absorption frequencies with the minor variation in the absorption peaks. Under the condition of $\varepsilon_{\mathrm{r}}$ set to 5.8, the lowest absorption peak is obtained at $1.9 \mathrm{GHz}$ whereas the minimum absorption peak is observed at $2.13 \mathrm{GHz}$ for the case of substrate relative permittivity set to 4.4. Fig. 5(a), represents the effect of variation in the dielectric loss tangent $(\tan \delta)$ from 0 to 1 on the absorption response. It is observed that the absorption bandwidth broadens with the subsequent deterioration in the absorption efficiency for larger $\tan \delta$ values. Lastly, the proposed MMA is studied for the variation in the number of unit cells with apparent result of total cell amount effect on the absorption frequency and efficiency as shown in Fig. 5(b). Hence, it can be concluded from the above numerical results that to obtain a wider absorption band, MMA has to be constructed on a thick substrate with the higher loss tangent. It is because of the increased imaginary part of MMA wave impedance with the consequence of reduction in the absorption efficiency, which can be improved by optimizing the geometrical parameters of the unit cell at the desired absorption frequency band. 


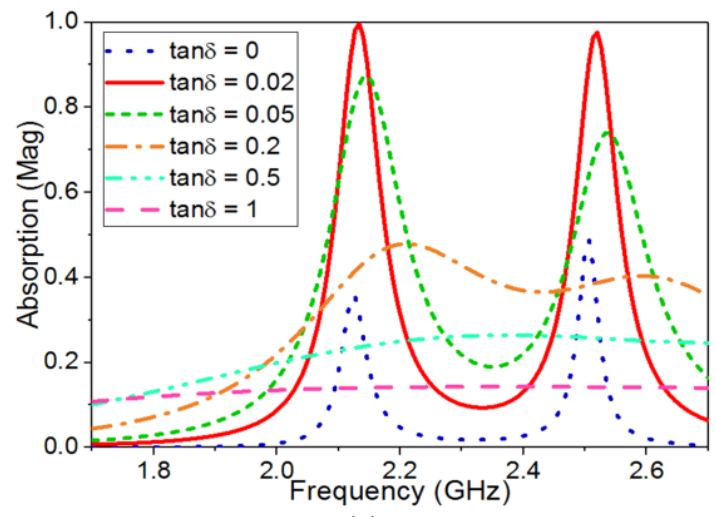

(a)

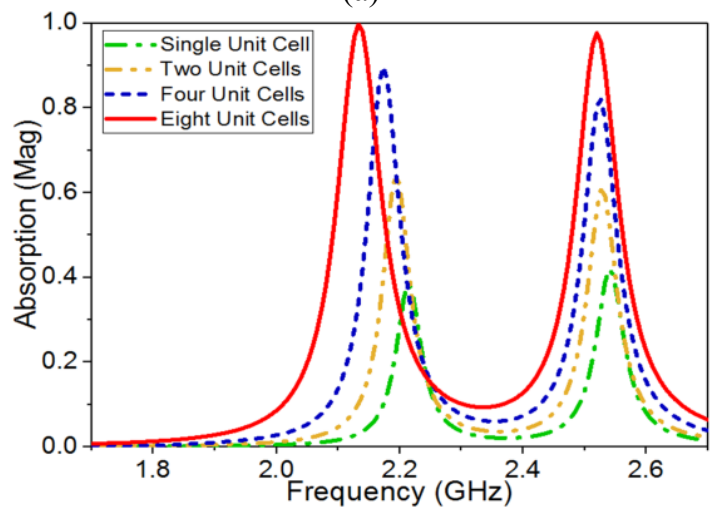

(b)

Figure 5: Simulated absorption response for various (a) Loss Tangents $(\tan \delta)$ and (b) Numbers of unit cells.
The comparison of the proposed absorber with the recently reported MTM based absorbers at the microwave frequencies is presented in Table 1. Table 1 illustrates that the proposed MMA has a more compact cell size in terms of guided wavelength at the lowest absorption frequency with a better absorption level and novelty in the unit cell geometry in addition to the promising feature of polarization independence, which has been assessed by the waveguide measurement technique.

\section{Measurement Setup and Experimental Results}

The proposed wide-angle ultrathin MMA is fabricated with $2 \times 4$ unit cell using standard printed circuit board $(\mathrm{PCB})$ technology with the prototype dimensions of $109.22 \mathrm{~mm} \times$ $54.61 \mathrm{~mm} \times 2.4 \mathrm{~mm}$ as shown in Fig. 1(b). Fig. 6 depicts the experimental setup of waveguide measurement technique.

In the experimental setup, WR-430 waveguide is connected to an Agilent N9912A vector network analyzer (VNA) via RG142 coaxial cable. Initially, a copper plate with the dimensions equivalent to that of the fabricated prototype is placed inside the waveguide. This copper plate is acting as an ideal reflector. Return loss from the ideal reflector has been measured. Subsequently, the fabricated prototype is placed in waveguide to measure the return loss for the calibration means. The measured return loss and computed absorption results of the proposed MMA prototype are represented in Fig. 7.

Table 1: Comparison of the proposed absorber with the state-of-the-art MTM absorbers at microwave frequencies

\begin{tabular}{|c|c|c|c|c|c|}
\hline MMA & $\begin{array}{c}\text { Unit Cell } \\
\text { Dimensions } \\
(\mathrm{mm})\end{array}$ & Absorption (\%) & $\begin{array}{l}\text { Frequencies } \\
\qquad(\mathrm{GHz})\end{array}$ & $\begin{array}{l}\text { Measurement } \\
\text { Method }\end{array}$ & $\begin{array}{l}\text { Polarization } \\
\text { Measurement }\end{array}$ \\
\hline [8] Dualband & $10 \times 10 \times 1$ & $99.8 \& 99.97$ & $5.5 \& 8.9$ & Free-space & Yes \\
\hline [9] Tripleband & $14 \times 14 \times 1$ & $99.9,97.9 \& 97.8$ & $\begin{array}{c}4.84,8.06 \& \\
11.28\end{array}$ & Free-space & Yes \\
\hline [11] Quadband & $20 \times 20 \times 0.8$ & $\begin{array}{c}98.5,97.7,94.8 \& \\
96\end{array}$ & $\begin{array}{c}4.34,6.68,8.58 \\
\& 10.64\end{array}$ & Free-space & No \\
\hline [12] Quadband & $3.33 \times 3.33 \times 0.217$ & $\begin{array}{c}99.47,99.94 \\
99.05 \& 99.55\end{array}$ & $\begin{array}{l}28.21,39.59 \\
52.78 \& 58.63\end{array}$ & - & No \\
\hline [13] Pentaband & $10 \times 10 \times 1$ & $\begin{array}{c}92.03,90.46,95.1 \\
91.65 \& 91.1\end{array}$ & $\begin{array}{c}5.28,7.36,9.52 \\
12.64 \& 16.32\end{array}$ & Free-space & Yes \\
\hline [17] Wideband & $12 \times 12 \times 3$ & $>90$ & 12.5 to 14 & Waveguide & No \\
\hline [19] Dualband & $12 \times 12 \times 0.9$ & $>95$ & $5.1 \& 8.5$ & Waveguide & No \\
\hline [20] Wideband & $9.5 \times 9.5 \times 3$ & $>90$ & 8.3 to 14.6 & Waveguide & No \\
\hline $\begin{array}{l}\text { Proposed } \\
\text { MMA }\end{array}$ & $\begin{array}{c}27.4 \times 27.4 \times \\
2.4\end{array}$ & $99.37 \& 97.18$ & $2.13 \& 2.52$ & Waveguide & Yes \\
\hline
\end{tabular}




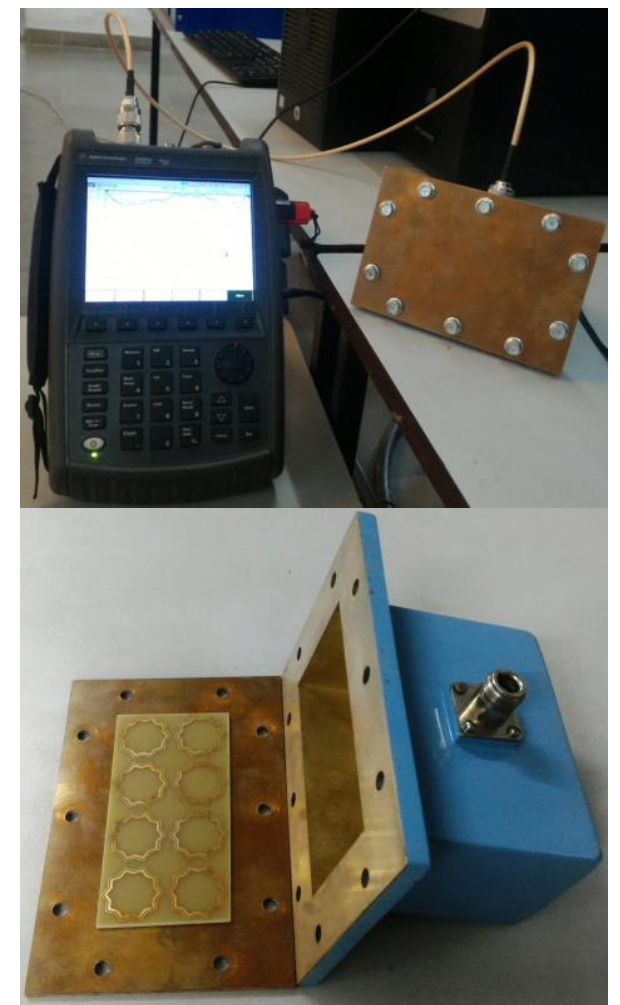

Figure 6: Experimental waveguide measurement arrangement.

The minimum return loss values are obtained at 2.114 $\mathrm{GHz}$ and $2.468 \mathrm{GHz}$ as shown in Fig. 7(a). The respective experimental value of absorption in comparison to simulated absorption is depicted in Fig. 7(b). The proposed MMA is experimentally verified for the polarization independence by setting different orientation angles for the concentric ten-point stars resonators, as shown in Fig. 1(b), to obtain the different polarization angles.

The $30^{\circ}$ and $45^{\circ}$ orientation angles are selected for the practical examination of polarization independence. Fig. 8 illustrates Sample-1 and Sample-2 corresponding to the inclination angle of electric field for the polarization angles of $30^{\circ}$ and $45^{\circ}$, respectively. The measured results of return loss and absorption response for Sample-1 and Sample-2 are depicted in Fig. 9. The return loss dips are obtained at the absorption frequencies of $2.109 \mathrm{GHz}$ and $2.472 \mathrm{GHz}$ for both Sample-1 and Sample-2. The numerically computed results and measured results for the oriented samples are in good agreement, verifying the polarization independence of MMA against varying polarization angles. However, the slight frequency shift in all measured results are marked which is mainly due to $\mathrm{TE}_{10}$ fundamental mode excitation inside the rectangular waveguide [17]. The frequency shift is about $0.03 \mathrm{GHz}$ to $0.05 \mathrm{GHz}$, which is possibly resulting from the fabrication tolerances of MMA prototype and the uncertainty in the material properties.

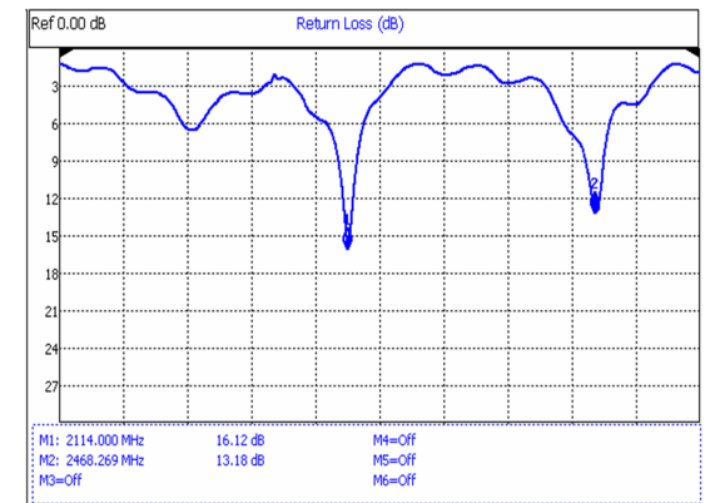

(a)

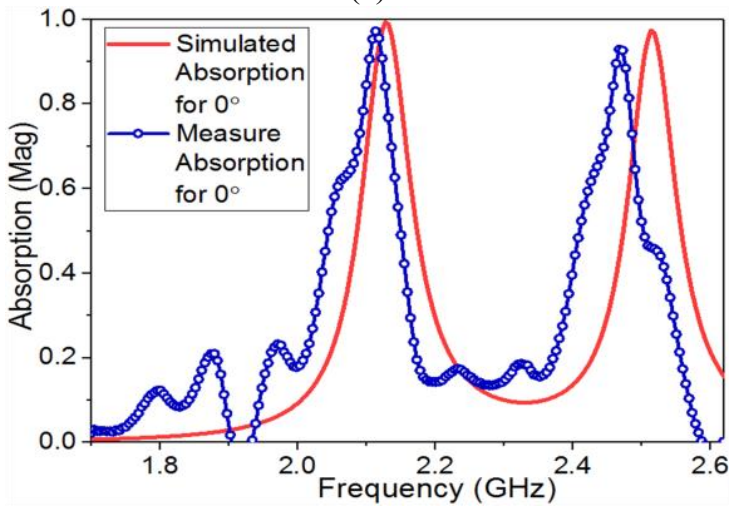

(b)

Figure 7: (a) Measured return loss and (b) computed absorption of proposed MMA.

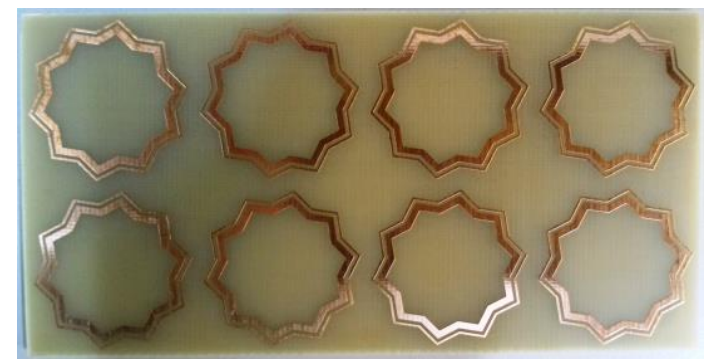

(a)

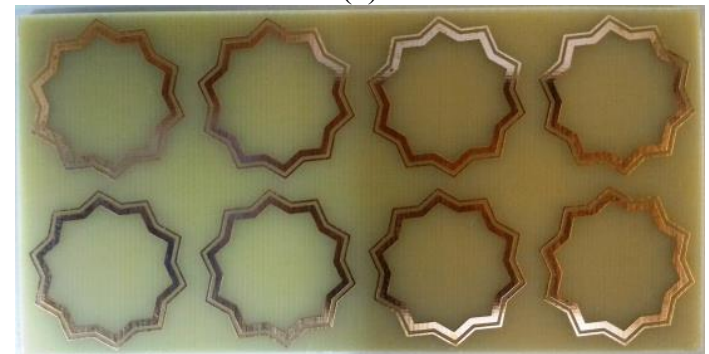

(b)

Figure 8: Photographs of fabricated prototypes with the orientation angles of: (a) $30^{\circ}$ (Sample-1) and (b) $45^{\circ}$ (Sample -2). 


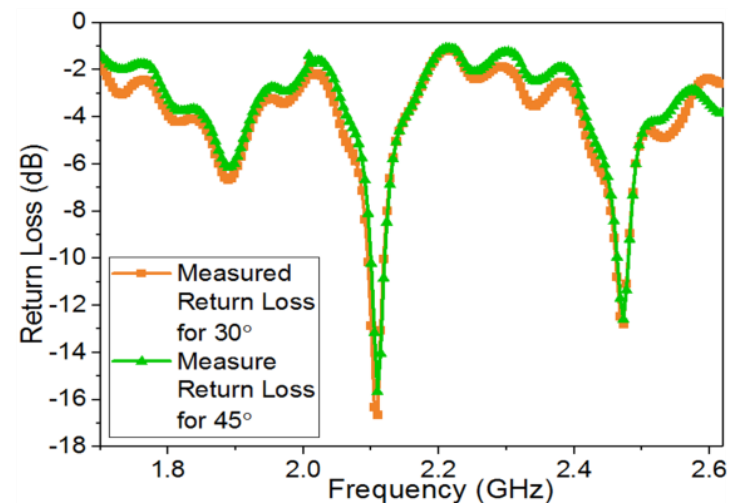

(a)

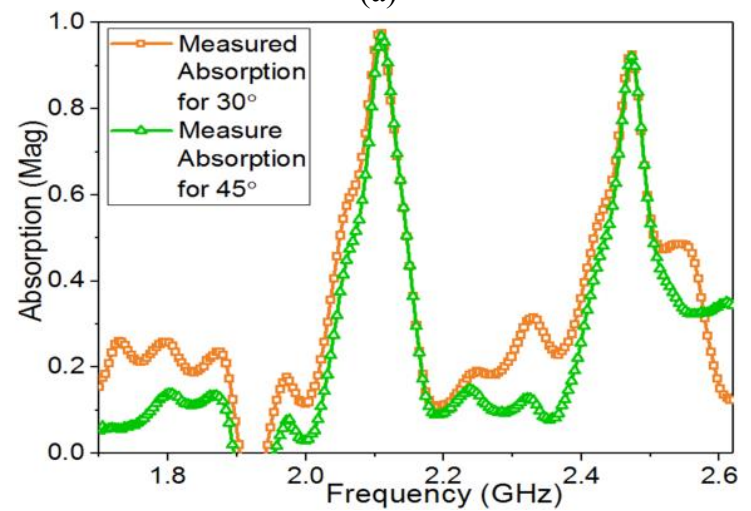

(b)

Figure 9: Measured results for sample-1 and sample-2: (a) return loss parameter and (b) absorption parameter.

\section{Conclusions}

The RF absorption performance of a wide-angle ultrathin microwave metamaterial absorber with the polarization independence has been evaluated in this article. The proposed MMA structure attains high absorption levels of $99.37 \%$ and $97.18 \%$ at $2.13 \mathrm{GH}$ and $2.52 \mathrm{GHz}$, respectively. The absorption response is studied for the variation of various electrical and geometrical parameters for the appropriate choice of unit cell design based on the requirement of bandwidth, frequency and absorption level. The proposed MMA has a wide incidence angle of about $60^{\circ}$ under TE mode and above $75^{\circ}$ for TM mode excitation with the high absorption value of $80 \%$ and $87 \%$, respectively. The proposed absorber has been also studied for the polarization independence and is verified practically using the waveguide measurement technique. For the practical demonstration of polarization independence, various MMA structures of different orientation angles are fabricated. The measured results show good agreement with the numerically computed results making proposed structure a good candidate for absorbing ambient UMTS band signals in RF energy harvesting and $\mathrm{S}$-band radar system applications. The proposed MMA unit cell is ultrathin with the thickness less than $\lambda / 56 \mathrm{~mm}$ at the lower absorption frequency.

\section{References}

1. V.G. Veselago, The electrodynamics of substances with simultaneously negative values of $\varepsilon$ and $\mu$, Soviet physics uspekhi 10: 509-514, 1968.

2. D.R. Smith, W.J. Padilla, D.C. Vier, S.C. NematNasser, S. Schultz, Composite medium with simultaneously negative permeability and permittivity, Physical review letters 84: 4184-4187, 2000.

3. R.A. Shelby, D.R. Smith, S. Schultz, Experimental verification of a negative index of refraction. Science 292: 77-79, 2001.

4. N.I. Landy, S. Sajuyigbe, J.J. Mock, D.R. Smith, W.J. Padilla, Perfect metamaterial absorber. Physical review letters 100: 207402, 2008.

5. F. Dincer, O. Akgol, M. Karaaslan, E. Unal, C. Sabah, Polarization angle independent perfect metamaterial absorbers for solar cell applications in the microwave, infrared, and visible regime, Progress In Electromagnetics Research 144: 93-101, 2014.

6. B.X. Wang, X. Zhai, G.Z. Wang, W. Q. Huang, L.L. Wang, Design of a four-band and polarizationinsensitive terahertz metamaterial absorber, IEEE Photonics Journal 7: 1-8, 2015.

7. P.V. Tuong, J.W. Park, J.Y. Rhee, K.W. Kim, W.H. Jang, H. Cheong, Y.P. Lee, Polarization-insensitive and polarization-controlled dual-band absorption in metamaterials, Applied Physics Letters 102: 081122, 2013.

8. S. Ramya, I.S. Rao, Design of polarization-insensitive dual band metamaterial absorber, Progress In Electromagnetics Research M 50: 23-31, 2016.

9. S. Bhattacharyya, S. Ghosh, K.V. Srivastava, Triple band polarization-independent metamaterial absorber with bandwidth enhancement at X-band, Journal of Applied Physics 114: 094514, 2013.

10. G.D. Wang, J.F. Chen, X. Hu, Z.Q. Chen, M. Liu, Polarization-insensitive triple-band microwave metamaterial absorber based on rotated square rings, Progress In Electromagnetics Research 145: 175-183, 2014.

11. M. Agarwal, A.K. Behera, M.K. Meshram, Wide-angle quad-band polarisation-insensitive metamaterial absorber, Electronics Letters 52: 340-342, 2016.

12. N. Wang, J. Tong, W. Zhou, W. Jiang, J. Li, X. Dong, $\mathrm{S}$. Hu, Novel quadruple-band microwave metamaterial absorber, IEEE Photonics Journal 7: 1-6, 2015.

13. D. Sood, C.C. Tripathi, A polarization insensitive compact ultrathin wide-angle penta-band metamaterial absorber, Journal of Electromagnetic Waves and Applications 31: 394-404, 2017.

14. M. Agarwal, A.K. Behera, M. K. Meshram, Dual resonating $\mathrm{C}$-band with enhanced bandwidth and broad X-band metamaterial absorber, Applied Physics A 122: 166,2016 
15. G. Sen, S.N. Islam, A. Banerjee, S. Das, Broadband Perfect Metamaterial Absorber on Thin Substrate for XBand and Ku-Band Applications, Progress In Electromagnetics Research C 73: 9-16, 2017.

16. W. Wang, Y. Chen, S. Yang, X. Zheng, Q. Cao, Design of a broadband electromagnetic wave absorber using a metamaterial technology, Journal of Electromagnetic Waves and Applications 29: 2080-2091, 2015.

17. H. Zhai, C. Zhan, L. Liu, Y. Zang, Reconfigurable wideband metamaterial absorber with wide angle and polarisation stability, Electronics Letters 51: 1624-1626, 2015.

18. D. Sood, C.C. Tripathi, A wideband wide-angle ultrathin low profile metamaterial microwave absorber, Microwave and Optical Technology Letters 58: 1131-1135, 2016 .

19. H. Zhai, C. Zhan, L. Liu, C. Liang, A new tunable dualband metamaterial absorber with wide-angle TE and TM polarization stability, Journal of Electromagnetic Waves and Applications 29: 774-785, 2015.

20. H. Zhai, B. Zhang, K. Zhang, C. Zhan, A stub-loaded reconfigurable broadband metamaterial absorber with wide-angle and polarization stability, Journal of Electromagnetic Waves and Applications 31: 447-459, 2017.

21. J. Sun, L. Liu, G. Dong, J. Zhou, An extremely broad band metamaterial absorber based on destructive interference, Optics Express 19: 21155-21162, 2011.

22. S. Ghosh, S. Bhattacharyya, D. Chaurasiya, K.V. Srivastava, Polarisation-insensitive and wide-angle multi-layer metamaterial absorber with variable bandwidths, Electronics Letters 51: 1050-1052, 2015.

23. M.R. Soheilifar, Design, fabrication, and characterization of scaled and stacked layers metamaterial absorber in microwave region, Microwave and Optical Technology Letters 58: 1187-1193, 2016.

24. Y. Liu, S. Gu, C. Luo, X. Zhao, Ultra-thin broadband metamaterial absorber, Applied Physics A 108: 19-24, 2012.

25. D. Chaurasiya, S. Ghosh, S. Bhattacharyya, K.V. Srivastava, An ultrathin quad-band polarizationinsensitive wide-angle metamaterial absorber, Microwave and Optical Technology Letters 57: 697-702, 2015.

26. S. Ramya, I.R. Srinivasa, A compact ultra-thin ultrawideband microwave metamaterial absorber, Microwave and Optical Technology Letters 59: 1837-1845, 2017.

27. M.D. Astorino, F. Frezza, N. Tedeschi, Broad-band terahertz metamaterial absorber with stacked electric ring resonators, Journal of Electromagnetic Waves and Applications 31: 727-739, . 2017

28. K.P. Kaur, T.K. Upadhyaya, M. Palandoken, Dual-Band Polarization-Insensitive Metamaterial Inspired Microwave Absorber for LTE-Band
Applications, Progress In Electromagnetics Research C 77: 91-100, 2017. 